Meta

Journal des traducteurs

Translators' Journal

\title{
Terminologies scientifiques et techniques en langue portugaise : projet de recherche
}

\section{José de Azevedo Ferreira}

Volume 32, numéro 3, septembre 1987

La fertilisation terminologique dans les langues romanes

URI : https://id.erudit.org/iderudit/001929ar

DOI : https://doi.org/10.7202/001929ar

Aller au sommaire du numéro

Éditeur(s)

Les Presses de l'Université de Montréal

ISSN

0026-0452 (imprimé)

1492-1421 (numérique)

Découvrir la revue

Citer cet article

de Azevedo Ferreira, J. (1987). Terminologies scientifiques et techniques en langue portugaise : projet de recherche. Meta, 32(3), 292-294.

https://doi.org/10.7202/001929ar d'utilisation que vous pouvez consulter en ligne. 


\section{TERMINOLOGIES SCIENTIFIQUES ET TECHNIQUES EN LANGUE PORTUGAISE : PROJET DE RECHERCHE}

JosÉ DE AZEVEDo FERREIRA

Universidade do Minho, Centro de Estudos Portugueses, Portugal

Ce n'est pas, à proprement parler, une communication que je vous présente, mais plutôt un projet de recherche ${ }^{1}$ que le Centre d'études portugaises de l'Université de Minho a soumis à l'appréciation de la JNICT dont nous attendons encore une réponse. Cette présentation a pour but de recueillir les réactions et d'éventuelles remarques et suggestions.

\section{LE PROJET}

On assiste aujourd'hui à une transformation extrêmement rapide de plusieurs champs du savoir scientifique et technologique qui entraîne un vieillissement des terminologies scientifiques et techniques et, en même temps, fait naître un grand nombre de vocables nouveaux, fondamentalement de la langue anglaise, dont l'adaptation correcte, du point de vue phonologique, morphologique et sémantique, à d'autres langues, est très souvent difficile. Or, ce manque d'une terminologie correcte et, en conséquence, l'imprécision et les lacunes terminologiques dans plusieurs domaines scientifiques, constituent des facteurs fortement négatifs dans certaines activités, telles que l'enseignement et la recherche.

Ces problèmes se font particulièrement sentir dans la langue portugaise qui n'a pas été protégée par une politique de défense et illustration de la langue, qui se justifie pleinement en vue de l'importance qu'elle commence à prendre dans le contexte mondial. Or, comme la langue portugaise a subi une véritable invasion, dans certains domaines scientifiques, tels que l'informatique, les sciences de l'éducation, les sciences de la terre, la biologie, etc., qui se traduit par une terminologie étrangère, avec prédominance anglaise, on sent un besoin absolu et urgent de la défendre, surtout si nous pensons à l'énorme espace géographique - un septième de la surface de la terre - où la langue portugaise est implantée : le Brésil et les pays africains d'expression officielle portugaise, en particulier l'Angola et le Mozambique. Il faut donc préserver ce riche instrument de communication d'environ 170 millions de sujets parlants, se fondant sur l'homogénéité des terminologies scientifiques et techniques, qui aura des conséquences bénéfiques dans la recherche et l'enseignement, rendant plus facile la circulation de publications et les échanges entre le Portugal, le Brésil et les pays africains, de professeurs, chercheurs et élèves.

En outre, plusieurs organismes publics et privés ont fréquemment manifesté le besoin de posséder une terminologie portugaise scientifiquement valable. Et ce besoin est de plus en plus urgent après la publication de la législation exigeant la traduction en portugais des noms de tous les produits étrangers avant d'être mis en vente. 
Pour mener à bien ce projet, les chercheurs du Centre d'études portugaises travailleront en étroite collaboration avec les équipes d'autres domaines scientifiques, en tenant compte des conditions très favorables existant à l'Université de Minho.

\section{STRATÉGIE}

L'équipe de chercheurs étudiera la méthodologie la plus adéquate à suivre, fera un inventaire de tous les travaux individuels (et ils sont nombreux) concernant le domaine de la terminologie linguistique portugaise. Il sera élaboré un inventaire des ouvrages étrangers qui abordent cette même thématique. Ensuite on entamera les contacts nécessaires avec les institutions ou les personnes qui, dans l'espace lusophone, en particulier le Brésil, travaillent sur des projets de recherche semblables ; de même, on doit se renseigner sur ce qui se passe dans les pays de langue romane qui ont déjà élaboré des projets de recherche de la même nature. Et nous pensons particulièrement aux travaux déjà réalisés ou en cours de réalisation par des institutions françaises, telles que le CILF, le CIREEL et FRANTERM. Les problèmes et les obstacles que la langue française a dû vaincre dans ce domaine pourront et devront aider fortement la langue portugaise, au fond, ils seront presque les mêmes.

On a même suggéré que, pendant la première année du projet — période décisive pour l'établissement des méthodes et des techniques d'organisation de la recherche l'équipe du Centre d'études portugaises pourrait compter sur la collaboration d'un linguiste français spécialiste dans ce domaine d'études sur les terminologies scientifiques et techniques. Ce linguiste, qui se déplacerait trois fois au cours de l'année, pendant des périodes de courte durée, constituerait un lien privilégié avec les institutions françaises cidessus mentionnées.

Dès le début des travaux on aura l'appui effectif du Centre de calcul de l'Université de Minho - Centre de Ciências e Engenharia de Sistemas -, avec lequel sera établi un accord de coopération. Ce Centre est déjà familiarisé avec ce type de travaux, puisqu'on y recourt fréquemment pour l'élaboration d'index de formes, de concordances, etc.

Dans la première phase du projet - qui durera deux ans - ses domaines d'incidence et, par conséquent, ses objectifs doivent être soigneusement délimités. Ainsi, en tenant compte de son importance dans le cadre du système d'enseignement et de son impact dans l'usage linguistique de beaucoup de sujets parlants, on a proposé que, dans cette première phase, soient réalisées des études sur les terminologies des domaines scientifiques suivants : $a$ ) sciences de l'éducation, $b$ ) informatique.

Ces études doivent conduire naturellement à la publication des vocabulaires respectifs.

L'organisation et le développement de ces études seront fondés sur un travail de nature interdisciplinaire devant être réalisé par les chercheurs du CEP (Centre d'études portugaises), du CEEDC (Centre d'études éducationnelles et développement communautaire) et le CCES (Centre d'informatique).

Dans une seconde phase du projet, on pourra déjà élargir l'étude des terminologies à d'autres domaines : $a$ ) sciences humaines, $b$ ) chimie et sciences de la terre, $c$ ) technologie textile.

La coordination de ce projet sera assurée par le président du CEP.

Nous faisons remarquer que c'est la première fois qu'un projet ${ }^{2}$ de cette nature et de cette portée est soumis aux responsables gouvernementaux, qui, malgré les moyens financiers et humains considérables mis en jeu, seront favorables au soutien nécessaire à ce projet. Car, finalement, les responsables politiques sont persuadés que les investissements dans la langue et la culture sont hautement rentables. Voici pourquoi, on assiste, depuis quelques jours à la promulgation d'un ensemble de mesures qui visent à la pro- 
tection de la langue. Ainsi, le 9 octobre 1985, le gouvernement a créé la CNALP (Commission nationale de la langue portugaise), un organe consultatif qui se propose de lui servir de conseiller dans la formulation et l'exécution d'une politique de la langue globale et cohérente, et dans toutes les questions concernant la défense et la diffusion de la langue portugaise. Pour présider cette commission le directeur de ce projet a été invité précisément.

D'un autre côté, nous rappelons que les grandes options du gouvernement pour les prochaines années, ce sont la langue, la culture et le patrimoine culturel.

C'est donc dans cette perspective optimiste en ce qui concerne la défense de la langue portugaise - le gouvernement a finalement pris conscience de son importance que nous attendons de la part des responsables un bon appui à la concrétisation de ce projet.

Notes

1. L'auteur et le directeur de ce projet est le président du Centre d'études portugaises, le Professeur Vitor Manuel de Aguiar e Silva.

2. Un projet a été présenté en 1983 par le Centre de linguistique de l'Université de Lisbonne, mais sa vie a été très courte. Cf. Maria Elisa Macedo de Oliveira (1986) : "Notícia sobre a elaboração da Terminologia Científica e Técnica da Língua Portuguesa ", in Revista do ICALP, nº 5, pp. 76-77. 